\title{
Heat conduction is a can of worms
}

Heat conduction is usually regarded as a phenomenon in which energy diffuses away from regions of high temperature, but diffusion may not always be a sufficient explanation.

WHETHER it is pleasing or otherwise to be told that a familiar principle is also a pack of lies is a matter of temperament. Some people are natural iconoclasts, others wish that at least some of the basic verities were eternal. But there are good reasons why what is known as Fourier's law (of heat conduction), which many people are still taught at high school, should be regarded as the crudest approximation to the truth. The interesting, but perplexing, question is that of what should take its place.

Most simply put, Fourier's law relates the flux of heat at any point in, say, a onedimensional solid such as a metal bar to the temperature gradient at that same point. The heat flux and temperature gradients are taken as proportional to each other, with a constant of proportionality called thermal conductivity and with a minus sign (to allow that heat flows against the temperature gradient). The relationship is that of the diffusion equation, describing the diffusion of a chemical in a liquid.

High-school problem-solvers know how this can be used to calculate the change of temperature with time at any point in, say, a conducting bar. The rate of change with time of the temperature at any point, determined by the rate at which energy accumulates there, is evidently proportional to the rate of change with distance of the heat flux, with a constant which is the reciprocal of the specific heat and with another minus sign. The outcome is an equation relating the first time-derivative of the temperature and the second distancederivative of the temperature. Given the distribution of temperature at the outset, it is then possible to calculate the temperature everywhere at all later times.

One might, for example, start with a metal bar at room temperature for half its length and at some higher temperature for the other half and follow the approach to equilibrium, when the temperature along the bar will be everywhere the same. But there is one obvious respect in which this result flies in the face of common sense: all solutions of these equations entail that the temperature everywhere along the bar will begin to change from the outset, however long the bar.

The implication is that the influence of the hotter half of the bar propagates instantaneously even to the most distant parts of the cooler half, which is unphysical. It is not merely that relativity disallows influences propagating faster than the speed of light, but that heat must get from one end of the bar to the other by some physical process plainly overlooked by Fourier's law.

This is the starting point for a review earlier this year by D. D. Joseph of the University of Minnesota and Luigi Preziosi of the University of Naples (Rev. Mod. Phys. 61, 41; 1989). Their objective is to define the circumstances in which heat can be propagated by means of waves, which has been observed in liquid helium and in some dielectric crystals at low temperatures. But their review inevitably begins with a discussion of the mechanisms of heat conduction and turns out to be a stimulating history of attempts to improve on Fourier's law, with the loose ends left dangling for all to see.

That there should be loose ends is not surprising; the mechanisms of heat transport are many and different. In a gas, energy is physically carried by molecules and transferred whenever they collide. In solids, on the other hand, lattice vibrations are a universal means of heat transport, but free electrons also play an important part in metals.

In all cases, the physical transport of heat from one place to another requires that some physical interaction should release energy at the destination - in a gas, the collision of an energetic molecule or, in a solid, the conversion of a quantum of energy in one vibrational mode into one or more quanta tied to other modes (which is possible only because of the anharmonicity of the vibrations of real crystals). Improvements on Fourier's law must take account of these processes, which involve microscopic energy conversion with characteristic times and distances (the time between collisions and the mean free path in a gas, for example).

Nobody will be surprised that James Clerk Maxwell is credited with the first important contribution to the argument, in 1867 (Phil. Trans. Roy. Soc. Lond. 157, $49 ; 1867)$. Following logic and his instincts, Maxwell (working with his kinetic theory) concluded that the spatial gradient of temperature is not simply related to the heat flux, but to a combination of that and a quantity proportional to the time-rate of change of the heat flux. The constant of proportionality must evidently have the dimensions of time; numerically, it is a 'relaxation time' related (in Maxwell's case) to the interval between molecular collisions.

Joseph and Preziosi observe that Max- well, having arrived at this conclusion, promptly discarded the extra term he had imported into the equation of Fourier's law on the grounds that the relaxation time must be negligible. In the event, his equation was rediscovered only in 1948 , by C. Cattaneo. Mathematically, Fourier's law in its simple form boils down to a differential equation for the temperature (as a function of position and time) which is strictly equivalent to the diffusion equation (which is why the ratio of the thermal conductivity and the specific heat of a material is called its thermal diffusivity). Maxwell's formulation, and that of Cattaneo, are by contrast equivalent to the partial differential equation known as the 'telegraph equation', which is itself a modification of the more familiar wave equation and whose solutions are waves which are attenuated as they propagate. So why do we not more often encounter phenomena in which heat seems to propagate as waves, not by diffusion?

As early as 1917, Nernst was arguing intuitively that, at low temperature, propagating heat should have inertia of a kind; a pulse of internal energy arriving at some point in one direction might be expected to continue in the same direction in the absence of the influences that, at high temperature, would randomize the internal motions. That, said Nernst, should make wave propagation possible. In 1941, Landau developed the two-fluid theory of liquid helium II and predicted the existence of heat waves, recognizable as temperature oscillations, which he called 'second sound'. The prediction was confirmed in 1944, when Peshkov measured the speed of second sound in helium II. Peshkov also predicted wavelike heat propagation in low-temperature crystals, also now confirmed.

So why not room-temperature heat waves? Joseph and Preziosi take the view that the question cannot be decided until more is understood about the spread of relaxation times in the process of heat conduction. Meanwhile, they advocate a further modification of the equation corresponding to Fourier's law to take account of these refinements. Luckily, as their history of the field shows, all kinds of new techniques - molecular dynamics, for example - are now being brought to bear on the issue. And, for practical purposes, Maxwell's guess that the simple form of Fourier's law suffices for most purposes remains valid. John Maddox 